\title{
ESTUDIO ESTADÍSTICO DE LA VARIABILIDAD DEL SILICATO EN EL MEDITERRÁNEO OCCIDENTAL (GOLFO DE LEON Y MAR CATALÁN)
}

K. MUNIZ

Departamento de Oceanografia da UFPE, Recife, Brasil C. RUIZ DE VILLA Departament d'Estadística Facultat de Biologia Barcelona, España A CRUZADO

Centre d'Estudis Avançats de Blanes, Blanes, España.

\section{RESUMEN}

A partir de datos hidrográficos de 1979 a 1993, se ha realizado un estudio estadístico con las concentraciones del silicato, sobre la variabilidad espacial y temporal de este nutriente en el Golfo de León y Mar Catalán (12 campañas oceariográficas). De acuerdo con los resultados del modelo de regresión múltiple, se observa una relación significativa entre silicato y profundidad/salinidad. Datos de 6 campañas mostraran homogeneidad de las pendientes en las ecuaciones de regresión múltiple, indicando que el grado de variación del silicato con la salinidad y con la profundidad es igual, independiente del area y de la estación del año. Eso sugiere que la distribución del silicato en la capa intermedia esta significativamente relacionada con procesos como la mezcla de las masas de agua y la disolución de los caparazones de los organismos. A partir de estas pendientes, un modelo predictor general fue elaborado.

Palabras claves: silicato, nutriente, estudio estadistico, Mar Mediterráneo.

\section{ABSTRACT}

Statistical Study And Spatial And Temporal Silicate Variability In The Western Mediterranean Sea (Gulf Of Lions And Catalan Sea).

Historical nutrient and hydrographic data (from 1979 to 1993), are used to study decadal scale temporal, spatial variability and statistical silicate distribution, in the Gulf of Lions and Catalan Sea (12 cruises). Results from a linear least squares multiple regression model, indicate a significant relationship between silicate and pressure and salinity. Data from six cruises showed homogeneity of slopes, indicating equal degree of silicate concentration variability with salinity and depth, which is independent of the geographic location and season. This suggests that the silicate distribution in the intermediary level, is significantly related to processes such as water mass mixing and the dissolution of the skeletons of organisms. From those slopes, a general model was calculated.

Key words: Silicate, nutrient, statistical study, Mediterranean sea 


\section{INTRODUCCIÓN}

El óxido de Silicio es conocido por ser una parte esencial en las estructuras sólidas de los silicoflagelados, diatomeas, algunos radiolarios y esponjas. Además de ser un nutriente limitante, el silicio también considerado nutriente refractario, puede ser relacionado con metales como el zinc, que tiene semejanzas con su perfil vertical y puede ser usado para conocer la participación de los metales traza en los estágios de los ciclos biogeoquímicos. Aunque sea incorporado en las frústulas de los organismos y no en los tejidos y ser regenerado a través de la su disolución, su perfil en la columna de agua sigue la distribución típica de los nutrientes (CHESTER, 1989).

La variabilidad en las medidas de los métodos de análisis de los nutrientes, a veces es muy acentuada y trabajos de investigaciones son dedicados exclusivamente para minimizar esta variabilidad. De acuerdo con WERNIMONT (1985), el problema principal sería determinar que variabilidad debe de ser minimizada. Hay dos métodos que pueden minimizar la variabilidad debido a errores experimentales, el directo y el estadístico. El primero incluye el método de agrupamientos en unidades experimentales dentro de bloques homogéneos, aumentando las condiciones de uniformidad sobre el cual el experimento está siendo realizado, mejorando de esta manera la exactitud de las medidas. El control indirecto (o estadístico) puede aumentar la precisión del experimento y eliminar fuentes potenciales de sesgos.

WERNIMONT (1985), presentan un esquema del uso de la regresión lineal en experimentos para estudiar la precisión y exactitud de las medidas, de manera a separar los errores y detectar las causas de la variabilidad, como también el control y minimización de los sesgos, mostrando una estimación de las limitaciones del método analítico. Otros autores han hechos trabajos usando técnicas estadísticas y determinando relaciones entre los diversos parámetros oceanográficos: EPPLEY et al., (1979) han usado el rango del nitrato de la relación densidad-nitrato para estimar la posible contribución del coeficiente de difusión horizontal al flujo del nitrato en el Sur de California; SMITH (1978), LEE et al.., (1981) y ATKINSON et al.., (1982) predijieron un plan de concentraciones de nutrientes, usando las relaciones de esos nutrientes con parámetros físico-químicos más facilmente detectables.

A partir de observaciones y estudios en el océano, hay un consenso general en oceanografia para asumir que la variación de algunos descriptores oceanográficos está explicada por la variación del parámetro más abundante. Para aquellos constituyentes que no se ajusten al modelo establecido, procesos especiales como influencia hidrotérmica o producción antropogénica son sienpre los responsables (MOORE, 1984). Si establecen relaciones entre distintos descriptores oceanográficos, y se consideran las distribuciones y relaciones patrones entre las propiedades conservativas y no conservativas, deberá tenerse en cuenta el ambiente dinámico y evolutivo del océano. 
El objetivo del presente trabajo, es estudiar la distribución del silicato en la columna de agua, en 12 campañas oceanográficas realizadas en el Mediterráneo Occidental (Mar Catalán y Golfo de Léon) entre 1979 y 1993. Se hace una selección de los muestreos que reflejan un menor error y se establecen relaciones entre los descriptores físicos de la columna de agua y su contenido en sílice inorgánica, con el fin de determinar las relaciones que expliquen el mayor porcentaje de variación de este nutriente én el Mediterráneo Occidental, al igual que su capacidad predictiva.

\section{MATERIAL Y MÉTODOS}

Se analizó estadísticamente la variación del silicato, salinidad, y profundidad en doce campañas oceanográficas realizadas en el Mediterráneo Occidental (tabla 1).

\section{Tabla 1 - Campañas Oceanográficas}

\begin{tabular}{|c|c|c|c|c|c|c|}
\hline$N^{0}$ & Campañas & Log.mín. & Log.máx. & Lat.mín. & Lat.máx. & Fecha \\
\hline \multicolumn{7}{|c|}{ G. de León } \\
\hline G1 & Pelagolion I & 3.08 & 5.88 & 42.05 & 43.46 & $12 / 09 / 86$ 3:00 \\
G2 & Pelagolion II & 3.12 & 5.85 & 42.05 & 43.45 & $15 / 12 / 866: 00$ \\
G3 & Discovery/88 & 3.61 & 6 & 40.95 & 43.38 & 15 a 24/12/88 \\
G4 & Bannock & 3.4 & 6.23 & 41.45 & 43.24 & $3 / 6$ a 11/7/89 \\
G5 & Rhodiber & 3.38 & 6.71 & 40.95 & 43.38 & 15 a 24/12/88 \\
G6 & Discovery/93 & 3.7 & 6.13 & 40.76 & 43.36 & $15 / 7$ a 2/8/93 \\
\hline \multicolumn{7}{|c|}{ Mar Catalán } \\
M1 & Tanit & 1.79 & 4 & 39.08 & 42.84 & $11 / 08 / 795: 00$ \\
M2 & Front 89 & 0.56 & 3.02 & 40.2 & 41.33 & $22 / 5$ a 13/6/89 \\
M3 & Front 90 & 2.13 & 3.17 & 40.14 & 41.3 & $21 / 02 / 90$ 9:00 \\
M4 & Front 91 & 2.15 & 3.07 & 40.51 & 41.31 & $21 / 04 / 9110: 00$ \\
M5 & Front 92 & 2.13 & 3.1 & 40.2 & 41.15 & $15 / 10$ a 3/11/92 \\
M6 & Front 93 & 0.03 & 3.43 & 38.8 & 41.61 & $29 / 06 / 931: 00$ \\
\hline
\end{tabular}

En todas las campañas se muestrearon en estaciones oceanográficas con botellas Niskin acopladas a la Rosette/CTD, determinándose de inmediato salinidad y temperatura. Los análisis de nutrientes también fueron realizados de inmediato con un autoanalizador Skalar. El método usado es basicamente el de MULLIN y RILEY (1955) y mide el silicato reactivo y no el polimerizado. El método está basado en la formación de complejos silicomolibidato, fosfomolibidato y arsenomolibidato y su posterior redución a una solución azul, en la cual se detecta la concentración del silicato a través de la colorimetría. El método tiene una precisión mayor que $0.1 \%$ y un rango de detección entre 0.1 a $140 \mu \mathrm{mol} / 1$ (PARSONS et al., 1984).

Con objeto de obtener una mayor uniformidad de los datos las campañas fueron divididas en tres capas distintas: 
$1^{\circ}$ capa superior - desde la superficie hasta la nutriclina (media $84.82 \mathrm{~m}$ - Golfo de León; 155.27 m - Mar Catalán).

$2^{\circ}$ capa intermedia - desde debajo de la nutriclina hasta la profundidad de salinidad máxima, que define la Masa de Agua Levantina (varia de $150 \mathrm{~m}$ a $689 \mathrm{~m}$ en el Golfo de Léon y de $79 \mathrm{~m}$ a $618 \mathrm{~m}$ en el Mar Catalán).

$3^{\circ}$ capa inferior - desde debajo de la profundidad de salinidad máxima hasta la última profundidad de muestreo.

La $1^{\circ}$ capa no ha sido todavia analizada al estar influenciada por varios procesos biológicos y químicos, lo que añade una mayor complejidad a su estudio. En general, los científicos interesados en la identificación de las masas de agua, evitan los primeros $200 \mathrm{~m}$ de la columna de agua, devido a la complejidad inerente a la capa del oceano afetada por la atmósfera y por la atividad biológica (KAMYKONOSKI y ZENTARA, 1986).

Siguiendo la metodologia de MONTGOMERY y PECK (1982), se utilizó el paquete estadístico BMDP (CALIFORNIA UNIVERSITY, 1990), para determinar las relaciones de regresión simple y múltiple entre el silicato (variable dependiente) y las otras variables. Dado que una correlación muy elevada entre las variables independientes en una regresión, puede afectar seriamente los resultados del análisis, se evaluar avaliar previamente el grado de la relación que existia (colinearidad). Para determinar el grado de colinearidad entre las variables consideradas independientes en las relaciones múltiples, se determinaron los VIFS (Variance Inflation Factors), que son los elementos de la diagonal de la matriz $\mathbf{C}=\left(\mathbf{X}^{\prime} \mathbf{X}\right)$, definido como $\mathbf{C j} \mathbf{j}=\left(\mathbf{1}-\mathbf{R}_{\mathbf{j}}{ }^{\mathbf{2}}\right)$, en donde $\mathbf{R}^{2}$ es el coeficiente de determinación obtenido cuando $x_{j}$ es correlacionado con las demás variables. Otro elemento determinado fue el "condition number" $k=\lambda_{\max } / \lambda_{\min }$ (valores proprios de la matriz $X X^{\prime}$ ) ( fuerte multicolinealidad - VIFS $>10$ y $k>100$ ).

Se obtuvieron las ecuaciones de regresión a partir de datos análogos entre las variables independientes profundidad y salinidad y la variable dependiente silicato, usando el análisis de covarianza para comparar estas relaciones funcionales de regresión entre las diversas campañas oceanográficas. Se determinó, por tanto, la homogeneidad entre las pendientes y entre las ordenadas en el origen, es decir, diferenciando entre las campañas cuyas relaciones perteneciesen a la misma población (a un nivel de significación $\alpha=0.05$ ).

A partir de las ecuaciones de regresión de las cuales se concluyó que era semejantes se obtuvo una ecuación general, que es considerada la predictora para todas las campañas. El criterio de elección de la relación predictora fue basado en los coeficientes de determinación $\left(R^{2}\right)$ (más elevados), en la raiz de los cuadrados medios de los residuales ( MS) (más bajos) y el grupo con mayor número de campañas con pendientes $y / 0$ ordenadas en la origen que no diferian significativamente.

Antes de hacer el estudio de la homogeneidad entre las pendientes y los interceptos, fueron extraidos los "outliers", de acuerdo con DRAPER y SMITH 
(1966). Los "outliers" solamente no fueron considerados, si presentaban sesgos contradictorios del punto de vista oceanográfico.

En la capa inferior $\left(3^{\circ}\right)$, las concentraciones de los nutrientes presentaban escasa variación, por lo que no mostraban relaciones de regresión significativas. Por tanto, se usó una ANOVA para comparar la distribución del silicato por debajo de la Masa de Agua Levantina entre las seguientes campañas oceanográficas: Pelagolion II, Discovery 88, Rhodiber, Discovery 93, Tanit, Fronts 89, Fronts 90, Fronts 93 (las demás no tenian resultados de la capa profunda). Dos rangos de profundidad fueron definidos: rango 1 de profundidades $<1000 \mathrm{~m}$, rango 2 de profundidades $1000 \mathrm{~m}$. Resultó por lo tanto una ANOVA con 3 factores $(2$ fijos = rangos de profundidades y campañas y 1 aleatorio= estaciones, anidado a las campañas), siguiendo la metodologia del paquete estadístico SAS (SAS Institute Inc., 1982), usando un análisis de varianza con datos no balanceados, procedimento GLM.

\section{RESULTADOS Y DISCUSIÓN}

Las ecuaciones de regresión simples y múltiple entre las variables de silicato (dependiente) y salinidad y profundidad (independientes), son determinadas en todas las campañas oceanográficas del Golfo de Leon y del Mar Catalán (Tablas 2 y 3). Los modelos de regresión múltiple presentan, en general, un coeficiente de determinación $\left(\mathbf{R}^{2}\right)$ mayor y un MS menor que los otros modelos de regresión simples. Es decir, la conjución de las variables indepedientes explica mejor la variación de la variable dependiente y hay una menor varianza de los datos en la relación. Sin embargo, en dos campañas (Front 90 y Front 91) los coeficientes de determinación no son significativos en esta relación.

Tabla 2. - Modelos de regresión múltiple, coeficiente de deterrminación $\left(\mathbf{R}^{2}\right)$ y raiz de los cuadrados medios de los residuales (MS) para cada campaña. Variable dependiente $y=$ silicatoy independientes $\mathrm{S}=$ salinidad y $\mathrm{P}=$ profundidad.

\begin{tabular}{|c|c|c|c|c|}
\hline Campaña & Estación & Ecuación & $\mathrm{MS}$ & $\mathrm{R}^{2}$ \\
\hline Pel.I & Ver/Otono & $\mathrm{y}=1.17 \mathrm{~S}+0.006 \mathrm{P}-43.39$ & 0.757 & 0.24 \\
\hline Pel.II & Invierno & $\mathrm{y}=6.10 \mathrm{~S}+0.007 \mathrm{P}-230.22$ & 0.365 & 0.94 \\
\hline Disc./88 & Invierno & $\mathrm{y}=12.2 \mathrm{~S}+0.003 \mathrm{P}-464.10$ & 0.562 & 0.69 \\
\hline Bannock & Verano & $\mathrm{y}=2.35 \mathrm{~S}+0.01 \mathrm{P}-87.19$ & 0.930 & 0.85 \\
\hline Rodiber & Ver/Otoño & $\mathrm{y}=2.76 \mathrm{~S}+0.004 \mathrm{P}-101.64$ & 1.053 & 0.37 \\
\hline Disc./93 & Verano & $\mathrm{y}=4.76 \mathrm{~S}+0.008 \mathrm{P}-179$ & 0.674 & 0.80 \\
\hline Tanit & Verano & $\mathrm{y}=-6.81 \mathrm{~S}+0.01 \mathrm{P}+263.03$ & 1.270 & 0.50 \\
\hline Front 89 & Primavera & $\mathrm{y}=5.46 \mathrm{~S}+0.005 \mathrm{P}-204.64$ & 0.706 & 0.80 \\
\hline Front 90 & Invierno & $\mathrm{y}=3.10 \mathrm{~S}+0.002 \mathrm{P}-112.75$ & 1.967 & 0.13 \\
\hline Front 91 & Primavera & $\mathrm{y}=2.71 \mathrm{~S}-0.005 \mathrm{P}-99.64$ & 1.139 & 0.13 \\
\hline Front 92 & Otoño & $\mathrm{y}=2.71 \mathrm{~S}+0.007 \mathrm{P}-101.58$ & 1.120 & 0.52 \\
\hline Front 93 & Verano & $\mathrm{y}=4.46 \mathrm{~S}+0.006 \mathrm{P}-168.09$ & 0.840 & 0.75 \\
\hline
\end{tabular}


Tabla 3. - Modelos de regresión simple, coeficiente de determinación (R2) y raiz de los cuadrados medios de los residuales (MS) para cada campaña. Variable dependiente silicato y independientes salinidad y profundidad.

\begin{tabular}{|c|c|c|c|c|c|c|}
\cline { 2 - 7 } \multicolumn{1}{c|}{} & \multicolumn{3}{|c|}{ Silicato/Salinidad } & \multicolumn{2}{c|}{ Silicato/Profundidad } \\
\hline$N^{\text {0 }}$ & Fcuación & R2 & MS & Fcuación & R2 & MS \\
\hline G1 & $Y=2.95-110.91$ & 0.16 & 0.786 & $y=0.007 x+1.61$ & 0.18 & 0.767 \\
G2 & $y=10.63 x-402.66$ & 0.71 & 0.751 & $y=0.01 x+2.99$ & 0.79 & 0.665 \\
G3 & $y=15.30 x-582.90$ & 0.62 & 0.605 & $y=0.006 x+4.13$ & 0.41 & 0.751 \\
G4 & $y=5.72 x-214.72$ & 0.72 & 1.916 & $y=0.02 x+2.21$ & 0.79 & 1.822 \\
G5 & $y=5.58 x-209.33$ & 0.3 & 1.101 & $y=0.007 x+3.75$ & 0.32 & 1.098 \\
G6 & $y=9.39 x-356.02$ & 0.69 & 0.824 & $y=0.01 x+2.06$ & 0.72 & 0.774 \\
M1 & $y=0.03 x+2.70$ & 0.38 & 1.206 & $y=0.003 x+3.36$ & 0.12 & 1.618 \\
M2 & $y=10.09 x-380.53$ & 0.62 & 0.956 & $y=0.008 x+3.69$ & 0.72 & 0.846 \\
M3 & $y=7.71 x-289.60$ & 0.19 & 1.962 & $y=0.003 x+5.66$ & 0.04 & 2.051 \\
M4 & $y=1.14 x-40.06$ & 0.02 & 1.191 & $y=-0.001 x+3.78$ & 0.01 & 1.271 \\
M5 & $y=10.11 x-384.25$ & 0.35 & 1.29 & $y=0.009 x+2.21$ & 0.49 & 1.135 \\
M6 & $y=8.07 x-305.03$ & 0.62 & 1.033 & $y=0.01 x+2.28$ & 0.66 & 0.987 \\
\hline
\end{tabular}

El estudio de la multicolinealidad entre salinidad y profundidad muestra que, el grado de covariación entre las dos variables independentes no afecta el modelo predictor de la variable dependiente silicato, ya que el VIF ( Variance Inflation Factors) no excede a 10 y el "condition number" es menor que 100 en todas las campañas, de acuerdo con MONTGOMERY y PECK (1976). Según estos autores una multicolinealidad significativa entre dos variables independientes, resulta en una mayor varianza de los cuadrados mínimos de los coeficientes de regresión, implicando en amplias diferencias en las estimas de los parámetros del modelo, entre diferentes muestras con el mismo nivel de $\mathrm{x}$, es decir, una menor precisión en la estimación de estos coeficientes.

Al comparar las ecuaciones de regresión entre todas las campañas oceanográficas, no se puede observar ningún grupo de campañas con ecuaciones (pendiente y ordenadas en el origen) que no diferen significantemente. Sin embargo, las relaciones presentan campañas con pendientes semejantes a un nível de significación $\alpha=0.05$ (Tablas 4 y 5 ). En las figuras 1 y 2 se puede observar las campañas con pendientes que no difieren significativamente en las relaciones silicato/profundidad y silicato/salinidad respectivamente.

De acuerdo con GARDENYES y BERNALDEZ (1970) en ecología a menudo se aplican las expresiones de regresión al análisis de medidas consideradas fuera de su contexto del espacio y tiempo., sin embargo, los sistemas de regresión son válidos en determinadas situaciones (localmente). Esto quiere 6 
decir que hay que tener en cuenta el espacio y el tiempo, por lo menos en determinadas relaciones de regresión, de lo contrario se perde una gran cantidad de información.

Analizando la relación silicato/profundidad, la no detección de similaridades entre las campañas es por supuesto debido a las variaciones temporales y espaciales existentes localmente.

Tabla 4 - Niveles de significación en la comparación de las ecuaciones de regresión simple de (variable dependente silicato, independentes salinidad y profundidad) de las campanias que no presentan diferenciss significativas a un nivel $\alpha=0.05$

\begin{tabular}{|c|c|c|c|c|c|c|c|c|c|c|}
\hline & \multicolumn{4}{|c|}{ Silicato/Salinidad } & \multicolumn{5}{|c|}{ Silicato/Profundidad } & \multirow[b]{2}{*}{$\mathrm{M}_{\mathrm{s}}$} \\
\hline & $\mathrm{G}_{2}$ & $\mathbf{G}_{6}$ & $\mathrm{M}_{2}$ & $\mathrm{M}_{3}$ & & $G_{1}$ & $\mathrm{G}_{3}$ & $G_{s}$ & $\mathbf{M}_{2}$ & \\
\hline$G_{2}$ & 1.00 & 0.47 & 0.78 & 0.85 & $\mathbf{G}_{1}$ & 1.00 & 0.84 & 0.97 & 0.34 & 0.34 \\
\hline $\mathrm{G}_{6}$ & 0.47 & 1.00 & 0.55 & 0.67 & $\mathrm{G}_{3}$ & 0.84 & 1.00 & 0.34 & 0.42 & 0.34 \\
\hline $\mathrm{M}_{2}$ & 0.78 & 0.55 & 1.00 & 0.99 & $\mathrm{G}_{3}$ & 0.97 & 0.34 & 1.00 & 0.14 & 0.23 \\
\hline $\mathrm{M}_{\mathrm{s}}$ & 0.85 & 0.67 & 0.99 & 1.00 & $\mathbf{M}_{2}$ & 0.34 & 0.42 & 0.14 & 1.00 & 0.92 \\
\hline & & & & & $\mathrm{M}_{\mathbf{3}}$ & 0.34 & 0.34 & 0.23 & 0.92 & 1.00 \\
\hline
\end{tabular}

La profundidad de la cambia en función de las condiciones del medio (turbidez, corrientes, etc...). Sin embargo, un grupo de campañas con pendientes iguales, muestra que el grado de variación, es decir, el gradiente y el flujo del silicato no varía significativamnete en el eje yertical con la localidad o de la estación del año, en profundidades distintas.

Añadiendo la salinidad como variable independiente, el modelo se vuelve más eficaz explicando mejor la variación del silicato en la columna de agua. Los coeficientes de determinación de una manera general son más elevados, los MS más bajos (Tablas 2 y 3) y un mayor número de campañas presentan pendientes que pertenecen a la misma población, con independencia del lugar y de la estación del año $\left(G_{2}, G_{5}, G_{6}\right.$ y $\left.M_{2}, M_{5}, M_{6}\right)$ (Tabla 5).

La explicación de esto es que el silicato siendo un nutriente refractário, puede seguir un patrón de comportamiento similar en determinadas áreas, pues su retorno como nutriente disuelto no se ve afectado significativamente por los distintos factores (CHESTER, 1990).

El modelo de regresión múltiple también sugiere la existencia de dos factores para explicar la variación del silicato relacionados entre sí, el proceso de disolución de los caparazones de los organismos (que está asociado a la profundidad) y la distribución de las masas de agua, definidas por la salinidad. 
Tabla 5. Niveles de significación en la comparación de las pendientes en las ecuaciones de regresión múltiple de las campañas que no presentan diferencias significativas a un nivel $\alpha=0.05$ ( $\mathrm{S}=$ salinidad y $\mathrm{P}=$ profundidad).

\begin{tabular}{|c|c|c|c|c|c|c|}
\hline & $\mathrm{G}_{2}$ & $\mathrm{G}_{3}$ & $\mathrm{G}_{6}$ & $\mathrm{M}_{2}$ & $\mathrm{M}_{3}$ & $\mathrm{M}_{6}$ \\
\hline $\mathrm{G}_{2}$ & $\begin{array}{l}S 1.00 \\
\text { P } 1.00 \\
\end{array}$ & $\begin{array}{l}\text { S } 0.21 \\
\text { P } 0.31 \\
\end{array}$ & $\begin{array}{l}\text { S } 0.46 \\
\text { P } 0.68 \\
\end{array}$ & $\begin{array}{l}\text { S } 0.72 \\
\text { P } 0.28 \\
\end{array}$ & $\begin{array}{l}\text { S } 0.29 \\
\text { P } 0.97 \\
\end{array}$ & $\begin{array}{l}\text { S } 0.43 \\
\text { P } 0.64 \\
\end{array}$ \\
\hline $\mathbf{G}_{s}$ & $\begin{array}{l}\text { S } 0.21 \\
\text { P } 0.31 \\
\end{array}$ & $\begin{array}{l}\mathrm{S} 1.00 \\
\mathrm{P} 1.00 \\
\end{array}$ & $\begin{array}{l}\text { S } 0.87 \\
\text { P } 0.09 \\
\end{array}$ & $\begin{array}{l}\text { S } 0.09 \\
\text { P } 0.53 \\
\end{array}$ & $\begin{array}{l}\text { S } 0.98 \\
\text { P } 0.16 \\
\end{array}$ & $\begin{array}{l}\text { S } 0.16 \\
\text { P } 0.19 \\
\end{array}$ \\
\hline $\mathbf{G}_{6}$ & $\begin{array}{l}\text { S } 0.46 \\
\text { P } 0.68 \\
\end{array}$ & $\begin{array}{l}\mathrm{S} 0.87 \\
\mathrm{P} 0.09 \\
\end{array}$ & $\begin{array}{l}S 1.00 \\
\text { P } 1.00 \\
\end{array}$ & $\begin{array}{l}S 0.60 \\
\text { P } 0.07 \\
\end{array}$ & $\begin{array}{l}\text { S } 0.34 \\
\text { P } 0.34 \\
\end{array}$ & $\begin{array}{l}\text { S } 0.81 \\
\text { P } 0.28 \\
\end{array}$ \\
\hline $\mathbf{M}_{2}$ & $\begin{array}{l}\text { S } 0.72 \\
\text { P } 0.28 \\
\end{array}$ & $\begin{array}{l}\mathrm{S} 0.09 \\
\mathrm{P} 0.53 \\
\end{array}$ & $\begin{array}{l}\text { S } 0.60 \\
P 0.07 \\
\end{array}$ & $\begin{array}{l}\mathrm{S} 1.00 \\
\mathrm{P} 1.00 \\
\end{array}$ & $\begin{array}{l}\text { S } 0.18 \\
\text { P } 0.19 \\
\end{array}$ & $\begin{array}{l}\text { S } 0.36 \\
\text { P } 0.34 \\
\end{array}$ \\
\hline $\mathrm{M}_{3}$ & $\begin{array}{l}\text { S } 0.29 \\
\text { P } 0.97 \\
\end{array}$ & $\begin{array}{l}S 0.29 \\
P 0.97 \\
\end{array}$ & $\begin{array}{l}S 0.34 \\
\text { P } 0.34 \\
\end{array}$ & $\begin{array}{l}\text { S } 0.18 \\
\text { P } 0.19 \\
\end{array}$ & $\begin{array}{l}S 1.00 \\
P 1.00 \\
\end{array}$ & $\begin{array}{l}\text { S } 0.32 \\
\text { P } 0.43 \\
\end{array}$ \\
\hline $\mathrm{M}_{6}$ & $\begin{array}{l}\text { S } 0.43 \\
\text { P } 0.64\end{array}$ & $\begin{array}{l}\text { S } 0.16 \\
\text { P } 0.19\end{array}$ & $\begin{array}{l}\text { S } 0.81 \\
\text { P } 0.28\end{array}$ & $\begin{array}{l}\text { S } 0.36 \\
\text { P } 0.34\end{array}$ & $\begin{array}{l}S 0.32 \\
\text { P } 0.43\end{array}$ & $\begin{array}{l}\text { S } 1.00 \\
\text { P } 1.00\end{array}$ \\
\hline
\end{tabular}

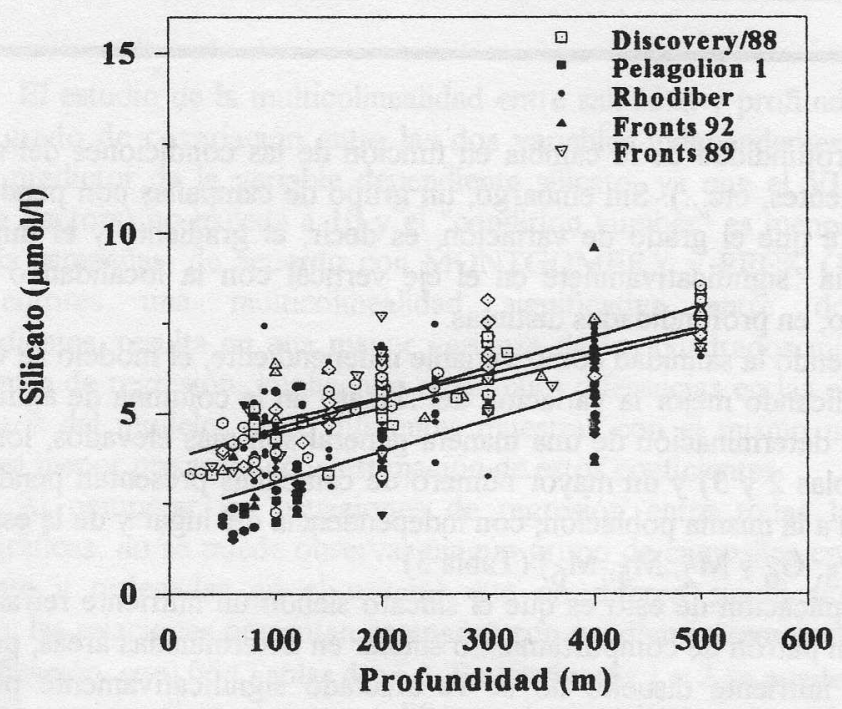

Fig. 1 Rectas de regresión de las campañas cuyas pendientes pertenecen a la misma población, en la relación silic./prof. 


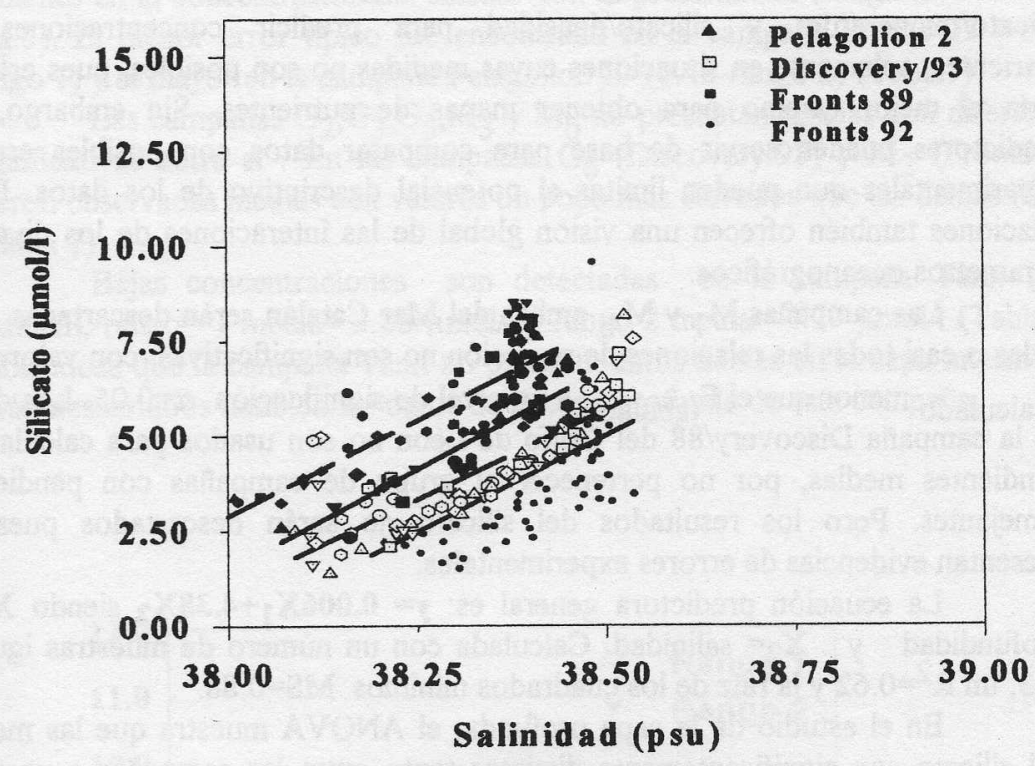

Fig. 2 Rectas de regresión de las campañas cuyas pendientes pertenecen a la misma población, en la relación sil./sal.

La tasa de disolución de la silice celular en la columna de agua, está asociada a varios factores químicos y biológicos y entre ellos al $\mathrm{pH}$, la temperatura y la presencia de aluminio, berilio, hierro, galio y titrio. Estos elementos a un $\mathrm{pH} 8$ pueden combinarse con el material celular y retardar la disolución de la sílice (RILEY Y SKIRROW, 1965). OKAMOTO et al. citados por RILEY y SKIRROW (1965), estudiaron la polimerización y despolimerización de la sílice coloidal a través de los iones hidróxidos y estos efectos dependen de la concentración de la sílice disuelta. De una manera general todos las factores que influyen en la disolución del sílice están asociados con la profundidad y las masas de agua y presentan pequeños rangos de variación. Cualquier variación debida a causas externas o a la propia estructura interna, puede resultar en cambios en las relaciones entre las variables conservativas y no conservativas en el mar.

De acuerdo con los resultados obtenidos, se calculó una pendiente media entre las campañas $G_{2}, G_{5}, G_{6}, M_{2}, M_{5}$ y $M_{6}$, cuyas pendientes pertenecen a la misma población, en la regresión múltiple con variables independientes profundidad y salinidad. Fue subtraído de los datos la media de cada variable en cada campaña, debido a que las ordenadas en la origen de estas ecuaciones son distintas. El objetivo es determinar una ecuación predictora para todas las 
campañas, con la recta de regresión pasando por el origen. KAMYKOWSKI y ZESNTARA (1986), usaron las ecuaciones de regresión cúbica de las relaciones silicato/temperatura y silicato/densidad para predicir concentraciones de nutrientes, solamente en situaciones cuyas medidas no son posibles, pues este no sería el mejor camino para obtener mapas de nutrientes. Sin embargo, los predictores pueden servir de base para comparar datos con posibles errores experimentales que pueden limitar el potencial descriptivo de los datos. Estas relaciones también ofrecen una visión global de las interaciones de los diversos parametros oceanográficos.

Las campañas $\mathrm{M}_{1}$ y $\mathrm{M}_{4}$, ambas del Mar Catalán serán descartadas pues todas o casi todas las relaciones de regresión no son significativas, con valores de $F_{\text {calculado }}$ menor que el $F_{\text {tabulado }}$ a un nivel de significación $\alpha=0.05$. Los datos de la campaña Discovery/88 del Golfo de Leon no son usados para calcular las pendientes medias, por no pertenecer al grupo de campañas con pendientes semejantes. Pero los resultados del silicato no serán descartados pues no presentan evidencias de errores experimentales.

La ecuación predictora general es: $\mathrm{y}=\mathbf{0 . 0 0 6 X _ { 1 }}+4.38 \mathrm{X}_{2}$ siendo $\mathrm{X}_{1}=$ profundidad y $\mathrm{X}_{2}=$ salinidad. Calculada con un número de muestras igual a 563 , un $\mathrm{R}^{2}=0.62$ y la raíz de los cuadrados mínimos $\mathrm{MS}=0.88$.

En el estudio de la capa profunda, el ANOVA muestra que las medias del silicato son significantemente distintas tanto entre las campañas a un nível $\alpha=0.05$, como también entre los rangos de profundidad. La similaridad o diferencia entre las campañas dos a dos, no depende de los rangos y viceversa, pues la interacción entre ambos no es significativa, con una probabilidad de $\mathrm{P}=0.548$ (Tabla 6).

Tabla 6 Análisis de Varianza (ANOVA) de las medias del silicato en la capa profunda. $\mathbf{C}^{\star} \mathbf{R}=$ interación campaña y rango, $\mathbf{E}\left(C^{*} \mathbf{R}\right)=$ Estación anidado a la interacción campaña y rango.

\begin{tabular}{|c|c|c|c|c|c|}
\hline Fuentes & DF & SS & MS & F & P \\
\hline Campaña & 7 & 165.21 & 23.60 & 33.80 & 0.000 \\
\hline Rango & 1 & 4.66 & 6.67 & 6.67 & 0.010 \\
\hline $\mathbf{C}^{*} \mathbf{R}$ & 5 & 2.80 & 0.80 & 0.80 & 0.548 \\
\hline $\mathbf{E}\left(\mathbf{C}^{*} \mathbf{R}\right)$ & 152 & 290.45 & 2.74 & 2.74 & 0.000 \\
\hline
\end{tabular}

Por tanto, la concentración del silicato aumenta con la profundidad en la capa abajo del Agua Levantina. Varios autores han discutido los datos de nutrientes en diversos océanos, concluyeron que el silicato tiene un comportamiento distinto al nitrato en relación a su redisolución en la columna de agua. Los compuestos de sílice además de ser refractários, parecen hundir mucho más rápido que el nitrógeno orgánico (FRIDERICH y CODISPOTI, 1979), y así su disolución es más lenta, provocando gradientes también en la capa profunda.

En el tabla 7 se pueden observar las medias balanceadas de las campañas en cada rango (LSM -media de los cuadrados mínimos), error típico de la media y 
los niveles de signifioación del ANOVA entre las medias de las campañas que no son significativamente distintas. Todas las campañas presentan un pequeño aumento en la concentración del silicato con la profundidad (rango $2>$ rango 1) (fig.3). El menor error típico fue encontrado en la campaña Fronts 93 ( 0.08 rango 1) y el mayor en la campaña Pelagolion II- (0.48-rango 2) (Tabla7).

Las campañas $\mathrm{G}_{2}, \mathrm{G}_{3}, \mathrm{G}_{5}, \mathrm{M}_{3}$ y $\mathrm{M}_{6}$ no presentan medias con diferencias significativas entre si . En las campañas $\mathrm{G}_{6}$ (Discovery/93) y $\mathbf{M}_{2}$ (Fronts 89) fueron observadas medias con valores un poco más elevados que las demás (fig. 3 y tabla 7).

Bajas concentraciones son detectadas en la campaña Tanit (Mar Catalán), rango $=1$ media $=3.88 \mu \mathrm{mol} / /$ y rango 2 media $=4.79 \mu \mathrm{mol} / /$ (Tabla 7 ). Sabiéndose que la campaña Tanit no presenta datos fiables en la capa intermedia, serán descartados también los datos de la capa profunda de esta campaña.

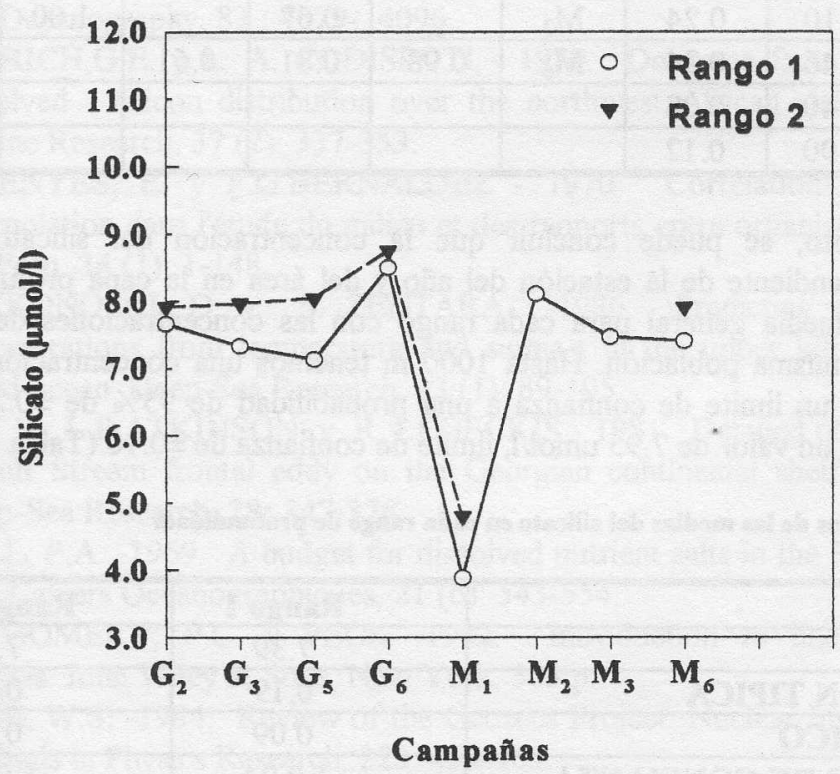

Fig. 3 Variación de la media de las concentraciones de silicato entre los rangos de profundidad de las campañas estudiadas.

De acuerdo con los resultados de 8 campañas estudiadas, 5 campañas no presentan medias con diferencias significativas en los respectivos rangos (Tabla 7). 
Tabla 7. - Medias balanceadas (LSM), error típico de la media(E) y niveles de significación (P) de la ANOVA de las campañas que no presentan diferencias en los respectivos rangos, a un nivel $\alpha=0.05$. $C=$ campanas $R=$ rango.

\begin{tabular}{|c|c|c|c|c|c|c|c|c|c|}
\hline $\mathbf{C}$ & $\mathbf{R}$ & $\mathbf{L S M}$ & $\mathbf{E}$ & & \multicolumn{3}{|c|}{$\mathbf{P}$} \\
\hline $\mathrm{G}_{2}$ & 1 & 7.66 & 0.25 & $\mathbf{R}_{\mathbf{1}}$ & $\mathrm{G}_{2}$ & $\mathrm{G}_{3}$ & $\mathrm{G}_{5}$ & $\mathbf{M}_{3}$ & $\mathbf{M}_{6}$ \\
\hline $\mathrm{G}_{2}$ & 2 & 7.91 & 0.48 & $\mathrm{G}_{2}$ & 1.00 & 0.21 & 0.06 & 0.57 & 0.32 \\
\hline $\mathrm{G}_{3}$ & 1 & 7.33 & 0.18 & $\mathrm{G}_{3}$ & 0.21 & 1.00 & 0.35 & 0.67 & 0.74 \\
\hline $\mathrm{G}_{3}$ & 2 & 7.96 & 0.21 & $\mathrm{G}_{5}$ & 0.06 & 0.35 & 1.00 & 0.22 & 0.07 \\
\hline $\mathrm{G}_{5}$ & 1 & 7.13 & 0.12 & $\mathbf{M}_{3}$ & 0.57 & 0.67 & 0.22 & 1.00 & 0.80 \\
\hline $\mathrm{G}_{5}$ & 2 & 8.04 & 0.24 & $\mathrm{M}_{6}$ & 0.32 & 0.74 & 0.07 & 0.80 & 1.00 \\
\hline $\mathrm{G}_{6}$ & 1 & 8.49 & 0.18 & $\mathbf{R}_{2}$ & $\mathrm{G}_{2}$ & $\mathrm{G}_{3}$ & $\mathrm{G}_{5}$ & $\mathbf{M}_{3}$ & $\mathbf{M}_{6}$ \\
\hline $\mathrm{G}_{6}$ & 2 & 8.75 & 0.18 & $\mathrm{G}_{2}$ & 1.00 & 0.93 & 0.81 & & 0.98 \\
\hline $\mathrm{M}_{1}$ & 1 & 3.88 & 0.34 & $\mathrm{G}_{3}$ & 0.93 & 1.00 & 0.81 & 0.67 & 0.81 \\
\hline $\mathrm{M}_{1}$ & 2 & 4.79 & 0.31 & $\mathrm{G}_{5}$ & 0.81 & 0.81 & 1.00 & & 0.61 \\
\hline $\mathrm{M}_{2}$ & 1 & 8.10 & 0.24 & $\mathrm{M}_{3}$ & & 0.67 & & 1.00 & \\
\hline $\mathrm{M}_{3}$ & 1 & 7.46 & 0.24 & $\mathrm{M}_{6}$ & 0.98 & 0.81 & 0.61 & & 1.00 \\
\hline $\mathrm{M}_{6}$ & 1 & 7.40 & 0.08 & & & & & & \\
\hline $\mathrm{M}_{6}$ & 2 & 7.90 & 0.12 & & & & & & \\
\hline
\end{tabular}

Por tanto, se puede concluir que la concentración del silicato es constante, independiente de la estación del año y del área en la capa profunda. Fue hecha una media general para cada rango con las concentraciones de las campañas de la misma población. Hasta $1000 \mathrm{~m}$ tenemos una concentración de 7.39 umol/ con un limite de confianza a una probabilidad de $95 \%$ de \pm 0.22 y abajo de $1000 \mathrm{~m}$ un valor de $7.95 \mathrm{umol} / \mathrm{h}$, limite de confianza de \pm 0.10 (Tabla 8).

Tabla 8 - Estadisticos de las medias del silicato en cada rango de profundidad.

\begin{tabular}{|l|c|c|}
\hline & Rango 1 & Rango 2 \\
\hline MEDIA & 7.39 & 7.95 \\
\hline DESVIACION TIPICA & 0.19 & 0.06 \\
\hline ERROR TIPICO & 0.09 & 0.03 \\
\hline $\begin{array}{l}\text { INTERVALO DE CONFIANZA } \\
(95 \%)\end{array}$ & \pm 0.24 & \pm 0.10 \\
\hline
\end{tabular}

Bethoux et al., (1992) han presentado valores de silicato en torno de $8.40 \mu \mathrm{mol} / \mathrm{l}$ en la capa profunda del Mediterraneo Occidental, en una latitud menor que $40^{\circ} \mathrm{N}$. Sin embargo, DELMAS y TREGNER (1984), han encontrado un valor medio de $7.5 \mu \mathrm{mol} / \mathrm{Kg}$ similar al encontrado en el presente trabajo. Esos autores comparando esta concentración con el valor encontrado en el Mediterraneo Oriental $(6.4 \mu \mathrm{mol} / \mathrm{/})$, concluyeran que en la parte oeste hay 1.2 
vezes más silicato que en la parte este, conclusión deducida anteriormente por McGILL (1969).

\section{REFERENCIAS}

ATKINSON, L. P., L. J. PIETRAFESA y E. E. HOFFMAN - 1982. An evaluation of nutrients Onslow Bay, North Carolina. Journal of Marine Research, 40: 347-699.

BETHOUX, J.P., P. MORIN, C. MADEC .y B. GENTILI - 1992. Phosphorus and nitrogen behaviour in the Mediterranean Sea. Deep-Sea Research, 29 (9) : 1641-1654.

CALIFORNIA UNIVERSITY -1990. BMDP Statistical Software Manual. N.J.Dixon. Berkeley, 1385 p.

HESTER R. - 1990. Marine Geochemistry. Unwin Hyman, London, 698 p.

DELMAS, R. y P. TREGNER -1984. Résultats de la Campagne PHYCEMED 2. Groupe Chimie des Ecosystèmes Marins, Contrast CNEXO 83/29000.

EPPLEY, R. W., E. H. RENGER y W.G. HARRISON -1979. Nitrate and phytoplankton production in southern California coastal waters. Limnology and Oceanography, $83: 4079-4096$.

FRIDERICH,G.E. y L. A. CODISPOTI - 1979. On some factors influencing dissolved silicon distribution over the northwest African shelf. Journal of Marine Research, 37 (2): 337-353.

GARDENYES, E. y F.G.BERNALDEZ - 1970. Corrélation regréssion et interpolation dans l'étude du milieu et des rapports entre organismes et milieu. Inv.Pesq.,34 (1): 1-148.

KAMYKONOSKI, D. y S. ZENTARA -1986. Predicting plant nutrient concentrations from temperature and sigma-t in the upper kilometer of the world ocean. Deep-Sea Research, 33 (1): 89-105.

LEE, T.N., L.P.ATKINSON y R. LEGECKIS - 1981. Detailed observations os a Gulf Stream frontal eddy on the Georgian continental shelf, April 1977. Deep-Sea Research, 28: 347-378.

McGILL, P.A. -1969. A budget for dissolved nutrient salts in the Mediterranean Sea. Cahiers Oceanographiques, 21 (6): 543-554.

MONTGOMERY, P.C. y PECK -1982. Introduction to linear regression analysis. John Wiley \& Sons, New York, 503 p.

MOORE, W.S. -1984. Review of the Geosecs Project. Nuclear Instruments and Methods in Physics Research, 223: 459-465.

MULLIN, J.B. y J. P.RILEY - 1955. Anal. Chim. Acta, 12: 162.

PARSONS, T.R., Y. MAITA y C. M. LALLI -1984. A manual of chemical and biological methods for seawater analysis. Pergamon Press, Oxford, $173 \mathrm{p}$.

RILEY, J.P. y G. SKIRROW - 1965. Chemical Oceanography. Academic Press. London, Volume I, $712 \mathrm{p}$.

SAS INSTITUTE INC. (eds) - 1982. SAS Users Guide: statistics. 1982 edition, Box 8000 , Cary, NC27511. 
SMITH -1978. Low-frequency fluxes of momentum, heat salt and nutrients at the edge of the Scotian shelf.Journal of Geophysical Research, 83: 4079-4096. WERNIMONT, G. T. -1985 . Use os Statistics to develop and evaluate analytical methods. Association of Official Analytical Chemists, USA, 183p.

Quadro 1. - Campañas Oceanográficas

\begin{tabular}{|c|c|c|c|c|c|c|}
\hline $\mathrm{N}^{\circ}$ & Campañas & Log.mín. & Log.máx. & Lat.mín. & Lat.máx. & Fecha \\
\hline $\mathrm{G}_{1}$ & Pelagolion I & 3.08 & 5.88 & 42.05 & 43.46 & 3 a $12 / 9 / 86$ \\
\hline $\mathrm{G}_{2}$ & Pelagolion II & 3.12 & 5.85 & 42.05 & 43.45 & 6 a $15 / 12 / 86$ \\
\hline $\mathrm{G}_{3}$ & Discovery/88 & 3.61 & 6.00 & 40.95 & 43.38 & 15 a 24/12/88 \\
\hline $\mathrm{G}_{4}$ & Bannock & 3.40 & 6.23 & 41.45 & 43.24 & $3 / 6$ a $11 / 7 / 89$ \\
\hline $\mathrm{G}_{5}$ & Rhodiber & 3.38 & 6.71 & 40.95 & 43.38 & 15 a $24 / 12 / 90$ \\
\hline $\mathrm{G}_{6}$ & Discovery/93 & 3.70 & 6.13 & 40.76 & 43.36 & $15 / 7$ a $2 / 8 / 93$ \\
\hline & Mar Catalán & & & & & \\
\hline $\mathrm{M}_{1}$ & Tanit & 1.79 & 4.00 & 39.08 & 42.84 & 5 a $11 / 8 / 79$ \\
\hline $\mathrm{M}_{2}$ & Front 89 & 0.56 & 3.02 & 40.20 & 41.33 & $22 / 5$ a $13 / 6 / 89$ \\
\hline $\mathrm{M}_{3}$ & Front 90 & 2.13 & 3.17 & 40.14 & 41.30 & 9 a 21/2/90 \\
\hline $\mathrm{M}_{4}$ & Front 91 & 2.15 & 3.07 & 40.51 & 41.31 & 10 a $21 / 4 / 91$ \\
\hline $\mathrm{M}_{5}$ & Front 92 & 2.13 & 3.10 & 40.20 & 41.15 & $15 / 10 \mathrm{a}$ \\
\hline $\mathrm{M}_{6}$ & Front 93 & 0.03 & 3.43 & 38.80 & 41.61 & $1 \mathrm{a} 29 / 6 / 93$ \\
\hline
\end{tabular}


Quadro 2. - Modelos de regresión múltiple, coeficiente de determinación $\left(\mathrm{R}^{2}\right)$ y raiz de los cuadrados medios de los residuales (MS) para cada campaña. Variable dependiente y=silicato y independientes $\mathrm{S}=$ salinidad y $\mathrm{P}=$ profundidad.

\begin{tabular}{|c|c|c|c|c|c|}
\hline Campaña & $\mathrm{N}^{\circ}$ & Estación & Ecuación & $\mathrm{MS}$ & $\mathrm{R}^{2}$ \\
\hline Pel.I & $\mathrm{G}_{1}$ & Ver/Otoño & $\mathrm{y}=1.17 \mathrm{~S}+0.006 \mathrm{P}-43.39$ & 0.757 & 0.24 \\
\hline Pel.II & $\mathrm{G}_{2}$ & Invierno & $\mathrm{y}=6.10 \mathrm{~S}+0.007 \mathrm{P}-230.22$ & 0.365 & 0.94 \\
\hline Disc./88 & $\mathrm{G}_{3}$ & Invierno & $\mathrm{y}=12.2 \mathrm{~S}+0.003 \mathrm{P}-464.10$ & 0.562 & 0.69 \\
\hline Bannock & $\mathrm{G}_{4}$ & Verano & $\mathrm{y}=2.35 \mathrm{~S}+0.01 \mathrm{P}-87.19$ & 0.930 & 0.85 \\
\hline Rodiber & $\mathrm{G}_{5}$ & Ver/Otoño & $\mathrm{y}=2.76 \mathrm{~S}+0.004 \mathrm{P}-101.64$ & 1.053 & 0.37 \\
\hline Disc./93 & $\mathrm{G}_{6}$ & Verano & $\mathrm{y}=4.76 \mathrm{~S}+0.008 \mathrm{P}-179$ & 0.674 & 0.80 \\
\hline Tanit & $\mathrm{M}_{1}$ & Verano & $\mathrm{y}=-6.81 \mathrm{~S}+0.01 \mathrm{P}+263.03$ & 1.270 & 0.50 \\
\hline Front 89 & $\mathrm{M}_{2}$ & Primavera & $\mathrm{y}=5.46 \mathrm{~S}+0.005 \mathrm{P}-204.64$ & 0.706 & 0.80 \\
\hline Front 90 & $\mathrm{M}_{3}$ & Invierno & $\mathrm{y}=3.10 \mathrm{~S}+0.002 \mathrm{P}-112.75$ & 1.967 & 0.13 \\
\hline Front 91 & $\mathrm{M}_{4}$ & Primavera & $\mathrm{y}=2.71 \mathrm{~S}-0.005 \mathrm{P}-99.64$ & 1.139 & 0.13 \\
\hline Front 92 & $\mathrm{M}_{5}$ & Otoño & $\mathrm{y}=2.71 \mathrm{~S}+0.007 \mathrm{P}-101.58$ & 1.120 & 0.52 \\
\hline Front 93 & $\mathrm{M}_{3}$ & Verano & $\mathrm{y}=4.46 \mathrm{~S}+0.006 \mathrm{P}-168.09$ & 0.840 & 0.75 \\
\hline
\end{tabular}

Quadro 3. - Modelos de regresión simple, coeficiente de determinación $\left(R^{2}\right)$ y raiz de los cuadrados medios de los residuales (MS) para cada campaña.

Silicato/Salinidad

\begin{tabular}{|c|c|c|c|c|c|c|}
\hline $\mathrm{N}^{\circ}$ & Ecuación & $\mathrm{R}^{2}$ & $\mathrm{MS}$ & Ecuación & $\mathrm{R}^{2}$ & $\mathrm{MS}$ \\
\hline $\mathrm{G}_{1}$ & $\mathrm{Y}=2.95-110.91$ & 0.16 & 0.786 & $\mathrm{y}=0.007 \mathrm{x}+1.61$ & 0.18 & 0.767 \\
\hline $\mathrm{G}_{2}$ & $\mathrm{y}=10.63 \mathrm{x}-402.66$ & 0.71 & 0.751 & $\mathrm{y}=0.01 \mathrm{x}+2.99$ & 0.79 & 0.665 \\
\hline $\mathrm{G}_{3}$ & $\mathrm{y}=15.30 \mathrm{x}-582.90$ & 0.62 & 0.605 & $\mathrm{y}=0.006 \mathrm{x}+4.13$ & 0.41 & 0.751 \\
\hline $\mathrm{G}_{4}$ & $\mathrm{y}=5.72 \mathrm{x}-214.72$ & 0.72 & 1.916 & $\mathrm{y}=0.02 \mathrm{x}+2.21$ & 0.79 & 1.822 \\
\hline $\mathrm{G}_{5}$ & $\mathrm{y}=5.58 \mathrm{x}-209.33$ & 0.30 & 1.101 & $\mathrm{y}=0.007 \mathrm{x}+3.75$ & 0.32 & 1.098 \\
\hline $\mathrm{G}_{6}$ & $\mathrm{y}=9.39 \mathrm{x}-356.02$ & 0.69 & 0.824 & $\mathrm{y}=0.01 \mathrm{x}+2.06$ & 0.72 & 0.774 \\
\hline $\mathrm{M}_{1}$ & $\mathrm{y}=0.03 \mathrm{x}+2.70$ & 0.38 & 1.206 & $\mathrm{y}=-0.003 \mathrm{x}+3.36$ & 0.12 & 1.618 \\
\hline $\mathrm{M}_{2}$ & $\mathrm{y}=10.09 \mathrm{x}-380.53$ & 0.62 & 0.956 & $\mathrm{y}=0.008 \mathrm{x}+3.69$ & 0.72 & 0.846 \\
\hline $\mathrm{M}_{3}$ & $\mathrm{y}=7.71 \mathrm{x}-289.60$ & 0.19 & 1.962 & $\mathrm{y}=0.003 \mathrm{x}+5.66$ & 0.04 & 2.051 \\
\hline $\mathrm{M}_{4}$ & $\mathrm{y}=1.14 \mathrm{x}-40.06$ & 0.02 & 1.191 & $\mathrm{y}=-0.001 \mathrm{x}+3.78$ & 0.01 & 1.271 \\
\hline $\mathrm{M}_{5}$ & $\mathrm{y}=10.11 \mathrm{x}-384.25$ & 0.35 & 1.290 & $\mathrm{y}=0.009 \mathrm{x}+2.21$ & 0.49 & 1.135 \\
\hline $\mathrm{M}_{6}$ & $\mathrm{y}=8.07 \mathrm{x}-305.03$ & 0.62 & 1.033 & $\mathrm{y}=0.01 \mathrm{x}+2.28$ & 0.66 & 0.987 \\
\hline
\end{tabular}


Quadro 4. - Niveles de significación en la comparación de las ecuaciones de regresión simple (variable dependiente silicato, independientes salinidad y profundidad) de las campañas con pendientes y interceptos que no presentan diferencias significativas a un nivel $=0.05$.

\begin{tabular}{|c|c|c|c|c|c|c|c|c|c|c|}
\hline \multicolumn{3}{|c|}{ Silicato/Salinidad } & \multicolumn{6}{c|}{ Silicato/Profundidad } \\
\hline \multirow{2}{||c|}{$\mathrm{G}_{2}$} & $\mathrm{G}_{6}$ & $\mathrm{M}_{2}$ & $\mathrm{M}_{5}$ & & $\mathrm{G}_{1}$ & $\mathrm{G}_{3}$ & $\mathrm{G}_{5}$ & $\mathrm{M}_{2}$ & $\mathrm{M}_{5}$ \\
\hline G6 & 1.00 & 0.47 & 0.78 & 0.85 & $\mathrm{G}_{1}$ & 1.00 & 0.84 & 0.97 & 0.34 & 0.34 \\
\hline M2 & 0.47 & 1.00 & 0.55 & 0.67 & $\mathrm{G}_{3}$ & 0.84 & 1.00 & 0.34 & 0.42 & 0.34 \\
\hline M5 & 0.78 & 0.55 & 1.00 & 0.99 & $\mathrm{G}_{5}$ & 0.97 & 0.34 & 1.00 & 0.14 & 0.23 \\
\hline & 0.85 & 0.67 & 0.99 & 1.00 & $\mathrm{M}_{2}$ & 0.34 & 0.42 & 0.14 & 1.00 & 0.92 \\
\hline
\end{tabular}

Quadro 5. - Niveles de significación en la comparación de las pendientes en las ecuaciones de regresión múltiple de las campañas que no presentan diferencias significativas a un nivel $=0.05$. $(\mathrm{S}=$ salinidad y $\mathrm{P}=$-profundidad $)$

\begin{tabular}{|c|c|c|c|c|c|c|}
\hline & $\mathrm{G}_{2}$ & $\mathrm{G}_{5}$ & $\mathrm{G}_{6}$ & $\mathbf{M}_{2}$ & $\mathrm{M}_{5}$ & $\mathrm{M}_{6}$ \\
\hline $\mathrm{G}_{2}$ & $\begin{array}{l}\text { S } 1.00 \\
\text { P } 1.00\end{array}$ & $\begin{array}{l}\text { S } 0.21 \\
\text { P } 0.31\end{array}$ & $\begin{array}{l}\text { S } 0.46 \\
\text { P } 0.68\end{array}$ & $\begin{array}{l}\text { S } 0.72 \\
\text { P } 0.28\end{array}$ & $\begin{array}{l}\text { S } 0.29 \\
\text { P } 0.97\end{array}$ & $\begin{array}{l}\text { S } 0.43 \\
\text { P } 0.64\end{array}$ \\
\hline $\mathrm{G}_{5}$ & $\begin{array}{l}\text { S } 0.21 \\
\text { P } 0.31\end{array}$ & $\begin{array}{l}\text { S } 1.00 \\
\text { P } 1.00\end{array}$ & $\begin{array}{l}\text { S } 0.87 \\
\text { P } 0.09\end{array}$ & $\begin{array}{l}\text { S } 0.09 \\
\text { P } 0.53\end{array}$ & $\begin{array}{l}\text { S } 0.98 \\
\text { P } 0.16\end{array}$ & $\begin{array}{l}\text { S } 0.16 \\
\text { P } 0.19\end{array}$ \\
\hline $\mathrm{G}_{6}$ & $\begin{array}{l}\text { S } 0.46 \\
\text { P } 0.68\end{array}$ & $\begin{array}{l}\text { S } 0.87 \\
\text { P } 0.09\end{array}$ & $\begin{array}{l}\text { S } 1.00 \\
\text { P } 1.00\end{array}$ & $\begin{array}{l}\text { S } 0.60 \\
\text { P } 0.07\end{array}$ & $\begin{array}{l}\text { S } 0.34 \\
\text { P } 0.34\end{array}$ & $\begin{array}{l}\text { S } 0.81 \\
\text { P } 0.28\end{array}$ \\
\hline $\mathrm{M}_{2}$ & $\begin{array}{l}\text { S } 0.72 \\
\text { P } 0.28\end{array}$ & $\begin{array}{l}\text { S } 0.09 \\
\text { P } 0.53\end{array}$ & $\begin{array}{l}\text { S } 0.60 \\
\text { P } 0.07\end{array}$ & $\begin{array}{l}\text { S } 1.00 \\
\text { P } 1.00\end{array}$ & $\begin{array}{l}\text { S } 0.18 \\
\text { P } 0.19\end{array}$ & $\begin{array}{l}\text { S } 0.36 \\
\text { P } 0.34\end{array}$ \\
\hline $\mathrm{M}_{5}$ & $\begin{array}{l}\text { S } 0.29 \\
\text { P } 0.97\end{array}$ & $\begin{array}{l}\text { S } 0.29 \\
\text { P } 0.97\end{array}$ & $\begin{array}{l}\text { S } 0.34 \\
\text { P } 0.34\end{array}$ & $\begin{array}{l}\text { S } 0.18 \\
\text { P } 0.19\end{array}$ & $\begin{array}{l}\text { S } 1.00 \\
\text { P } 1.00\end{array}$ & $\begin{array}{l}\text { S } 0.32 \\
\text { P } 0.43\end{array}$ \\
\hline $\mathrm{M}_{6}$ & $\begin{array}{l}\text { S } 0.43 \\
\text { P } 0.64\end{array}$ & $\begin{array}{l}\text { S } 0.16 \\
\text { P } 0.19\end{array}$ & $\begin{array}{l}\text { S } 0.81 \\
\text { P } 0.28\end{array}$ & $\begin{array}{l}\text { S } 0.36 \\
\text { P } 0.34\end{array}$ & $\begin{array}{l}\text { S } 0.32 \\
\text { P } 0.43\end{array}$ & $\begin{array}{l}\text { S } 1.00 \\
\text { P } 1.00\end{array}$ \\
\hline
\end{tabular}


Quadro 6. - Análisis de Varianza (ANOVA) de las medias del silicato en la capa profunda. $\mathrm{C}^{*} \mathrm{R}=$ interación campaña y rango, $\mathrm{E}(\mathrm{C} * \mathrm{R})=$ Estación anidado a la interacción campaña y rango.

\begin{tabular}{|c|c|c|c|c|c|}
\hline Fuentes & DF & SS & MS & F & P \\
\hline Campaña & 7 & 165.21 & 23.60 & 33.80 & 0.000 \\
\hline Rango & 1 & 4.66 & 6.67 & 6.67 & 0.010 \\
\hline C*R & 5 & 2.80 & 0.80 & 0.80 & 0.548 \\
\hline E(C*R) & 152 & 290.45 & 2.74 & 2.74 & 0.000 \\
\hline
\end{tabular}

Quadro 7. - Medias balanceadas (LSM), error típico de la media(E) y niveles de significación (P) del ANOVA de las campañas que no presentan diferencias en los respectivos rangos, a un nivel $=0.05$. $\mathrm{C}=$ campanias $\mathrm{R}=$ rango.

\begin{tabular}{|c|c|c|c|c|c|c|c|c|c|}
\hline $\mathrm{C}$ & $\mathrm{R}$ & $\mathrm{LSM}$ & $\mathrm{E}$ & \multicolumn{2}{|c|}{} & \multicolumn{2}{|c|}{$\mathrm{P}$} \\
\hline $\mathrm{G}_{2}$ & 1 & 7.66 & 0.25 & $\mathrm{R}=1$ & $\mathrm{G}_{2}$ & $\mathrm{G}_{3}$ & $\mathrm{G}_{5}$ & $\mathrm{M}_{3}$ & $\mathrm{M}_{6}$ \\
\hline $\mathrm{G}_{2}$ & 2 & 7.91 & 0.48 & $\mathrm{G}_{2}$ & 1.00 & 0.21 & 0.06 & 0.57 & 0.32 \\
\hline $\mathrm{G}_{3}$ & 1 & 7.33 & 0.18 & $\mathrm{G}_{3}$ & 0.21 & 1.00 & 0.35 & 0.67 & 0.74 \\
\hline $\mathrm{G}_{3}$ & 2 & 7.96 & 0.21 & $\mathrm{G}_{5}$ & 0.06 & 0.35 & 1.00 & 0.22 & 0.07 \\
\hline $\mathrm{G}_{5}$ & 1 & 7.13 & 0.12 & $\mathrm{M}_{3}$ & 0.57 & 0.67 & 0.22 & 1.00 & 0.80 \\
\hline $\mathrm{G}_{5}$ & 2 & 8.04 & 0.24 & $\mathrm{M}_{6}$ & 0.32 & 0.74 & 0.07 & 0.80 & 1.00 \\
\hline $\mathrm{G}_{6}$ & 1 & 8.49 & 0.18 & $\mathrm{R}_{2}$ & $\mathrm{G}_{2}$ & $\mathrm{G}_{3}$ & $\mathrm{G}_{5}$ & $\mathrm{M}_{3}$ & $\mathrm{M}_{6}$ \\
\hline $\mathrm{G}_{6}$ & 2 & 8.75 & 0.18 & $\mathrm{G}_{3}$ & 1.00 & 0.93 & 0.81 & & 0.98 \\
\hline $\mathrm{M}_{1}$ & 1 & 3.88 & 0.34 & $\mathrm{G}_{3}$ & 0.93 & 1.00 & 0.81 & 0.67 & 0.81 \\
\hline $\mathrm{M}_{1}$ & 2 & 4.79 & 0.31 & $\mathrm{G}_{5}$ & 0.81 & 0.81 & 1.00 & & 0.61 \\
\hline $\mathrm{M}_{2}$ & 1 & 8.10 & 0.24 & $\mathrm{M}_{3}$ & & 0.67 & & 1.00 & \\
\hline $\mathrm{M}_{3}$ & 1 & 7.46 & 0.24 & $\mathrm{M}_{6}$ & 0.98 & 0.81 & 0.61 & & 1.00 \\
\hline $\mathrm{M}_{6}$ & 1 & 7.40 & 0.08 & & & & & & \\
\hline $\mathrm{M}_{6}$ & 2 & 7.90 & 0.12 & & & & & & \\
\hline
\end{tabular}

Quadro 8. - Estadísticos de las medias del silicato en cada rango de profundidad.

\begin{tabular}{|l|c|c|}
\hline & Rango 1 & Rango 2 \\
\hline MEDIA & 7.39 & 7.95 \\
\hline DESVIACION TIPICA & 0.19 & 0.06 \\
\hline ERROR TIPICO & 0.09 & 0.03 \\
\hline INTERVALO DE CONFIANZA (95\%) & \pm 0.24 & \pm 0.10 \\
\hline
\end{tabular}

